\title{
Principal component analysis for characterising homogeneity in powder mixing using image processing techniques
}

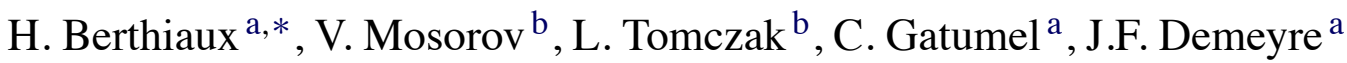 \\ a Laboratoire de Génie des Procédés des Solides Divisés, UMR CNRS 2392, Ecole des Mines d'Albi-Carmaux, \\ Campus Jarlard, Route de Teillet, 81000 ALBI, France \\ ${ }^{\mathrm{b}}$ Computer Engineering Department, Technical University of Lodz, 18/22 Stefanowskiego 90-924, Poland
}

\begin{abstract}
The many methods which exist to characterise the quality of a powder mixture have been recently reviewed and linked with mixing mechanisms in current literature. In this paper, we try to develop a novel methodology for defining and characterising homogeneity using principal component analysis (PCA) as an alternative to well-known statistical methods, such as auto-correlation functions or variances. We apply this to image analysis for the case of a powder mixture flowing out of a continuous mixing device. An emphasis is placed on the calculation in real-time of the degree of homogeneity of loose materials on the conveyor belt, carrying this mixture. Mass flow disturbances applied to a binary mixture are studied by the proposed methodology, which is found to be sensitive to small structural defaults.
\end{abstract}

Keywords: Powder mixing; Homogeneity criterion; Image analysis; Principal component analysis

\section{Introduction-state of the art}

Powder mixing is a widely used unit operation in the pharmaceutical, agro-food, cement or chemical industries. The process is complex as it depends on many factors operating at various scales: single particle properties, bulk particle properties, general mixer design and operation, combination of operating conditions, mixture formulation, etc. The quality of a mixture, which may be the degree of homogeneity of loose material at the outlet of a mixer, is important for end-user properties as perceived by customers and/or for in-process properties used by manufacturers for normative procedures. In addition, mixing process optimisation is a matter of reducing mixing time and saving electrical energy, especially for low added-value products.

The definition of mixture quality first requires the knowledge of the scale at which it has to be defined, that is the scale of scrutiny of a mixture. For example in the case of pharmaceutical tablet the size of scrutiny is probably equal to a tablet size, that

\footnotetext{
* Corresponding author.

E-mail address: berthiau@enstimac.fr (H. Berthiaux).
}

is to say the size of the patients dose. However, this may not be so when the patient interferes, such as when he is allowed to sample himself in the package (if the patient take a piece of tablet for example).

The many methodologies that exist to characterise mixture homogeneity and its structure were recently reviewed and linked with mixing mechanisms by Gyenis [1]. A distinction must be made between the techniques that calculate a structural criterion and those which generate an overall criterion, which are well known in industrial practice. The standard deviation of the composition of a certain number of samples, as well as the corresponding variance, coefficient of variation, or mixing indices belong to this latter category. Weinekotter [2] illustrated the limits of such analyses by discussing two continuous processes having the same variance but radically different organisation: (a) 12 samples with compositions alternating on both sides of the mean; (b) six samples of identical composition higher than the mean followed by six samples of identical composition lower than the mean. When compared to each other from a "customer" end-used property, it is obvious that process (b) is inadequate while it may satisfy a pharmaceutical quality criterion.

On the other hand, fractal analysis, phase-space techniques and the study of auto-correlation functions allows taking into 
account the structure of a powder mixture, as the relative positions of the samples are not destroyed by the analysis. Danckwerts [3] was probably the first to suggest the use of autocorrelation functions to describe the scale of segregation of a mixture, that is the maximum size of segregated regions in a mixture $\cdots$ or the sample size above which powder homogeneity is only due to random effects. The auto-correlation function $R$ characterises the interdependence of the compositions of any two samples of a series of samples, separated by a distance $r$ (or by a given number of samples). If no correlation exists, then the value of $R(r)$ is equal to zero and the mixture can be considered as homogeneous at this scale. In other words, the scale of segregation becomes equal to the scale of scrutiny. In practice, the first "distance" $r_{0}$ for which $R$ equals zero can be read from a plot of $R$ versus $r$, called a correlogram. This distance is the parameter for characterising the structure of the mixture, as it gives a measure of the maximum length of no correlation between samples. However, the determination of $r_{0}$ is not always easy, at least because a correlogram may exhibit asymptotic behaviour, or because it is very sensitive to a small variations in the composition of the samples. Indeed, there is still a need for developing new, meaningful, industry-pertinent methodologies for quantifying the homogeneity of powder mixtures.

From the point of view of the techniques used for obtaining such information, recent emphasis has been placed on on-line methods which avoid the interference of thief sample probes [4] and the related statistical problems. Optical measurement techniques have received wide attention in the scientific community, especially in the past decade, from the pioneering work of Harwood et al. [5], to that of Berntsson et al. [6] (one may also refer to Weinekötter and Reh [7] or Steinmetz et al. [8]). Near Infrared (NIR) spectroscopy is now beginning to be used in industrial R\&D for measuring powder blends. However, many of the workers involved in such projects find difficulties in the calibration procedures and time stability of the signals. Also concerning these type of methods, but very much at the research stage, we may cite laser induced fluorescence as a very promising tool for pharmaceutical mixtures [9]. Electrical capacitance tomography (ECT) has been under rapid development since the mid-nineties and has been applied to real cases in general powder technology [10-12], and in powder mixing in particular [13]. While it seems to be a valuable alternative to optical methods, it seems better suited for coherent pipe flows, that is to say with low disturbance of the flow rates because porosity changes have a strong influence on data reliability. In comparison to other measurement techniques, image analysis is non-destructive and is characterised by a great speed, which is very important in on-line systems. In the recent scientific literature, Muerza et al. [14] have proposed such a method. In this work the method is applied to two free-flow powders differing in colour mixed by a continuous static mixing unit and poured for transport by a belt conveyor without disturbance. A CCD camera placed on the conveying line captures images of the moving belt. These images are then treated and analysed using the auto-correlation technique to obtain the characteristic distance $r_{0}$. Because this required a critical analysis of the correlograms, with respect to their shape, it was performed off-line from the measurement chain.
From the above, one may understand that a single method cannot cover the whole range of possible powder blends. Also, when considering its applicability to industrial problems, the exactitude of the method comes after other type of considerations, such as the stability of the measurement with time, easy calibration procedures or no calibration required at all, sampling needed or not, compatibility with industrial conditions, real-time data analysis, .... Therefore, working on new on-line methodologies for accurate data treatment in real-time is still an important field of investigation. This paper proposes a novel method based on real-time principal component analysis of powder mixture images captured by a CCD camera with the claim that this methodology of data processing may be adapted to other types of on-line techniques, such as capacitance measurements or NIR spectroscopy.

\section{Powder mixture measurement based on image analysis}

The method developed in this paper aims to measure the degree of homogeneity of loose materials on a conveyor belt using image processing techniques. For this, one powder (semolina; particle size, 100-250 $\mu \mathrm{m}$ ) and a tracer (semolina coloured by iodine adsorption) were used as particulate systems having identical properties except colour. The measurement chain consists of a CCD camera placed over the conveyor belt which transports the mixture of the loose materials, as well as a $\mathrm{PC}$ computer. The schematic diagram of this system is presented in Fig. 1.

A time sequence of images is captured by the camera and represents the solid mixture on the belt during conveying. These images are treated with specially designed computer software to calculate the homogeneity ratio of the mixture. Each image represents a two-dimensional sample of analysed mixture. The pinhole camera model used (see Fig. 2) is detailed in Appendix and provides a continuous brightness function corresponding to each frame. Digitizing a frame consists in sampling and quantifying the function, it gives a grey scale image containing information about tracer concentration. Indeed the black tracer concentration is directly linked with the brightness as its reflectance is different from that of the white particles.

In the example shown on Fig. 3, a sample and a quantized binary mixture image is shown. The diameter $\phi$ of any particle in the mixture is approximately equal to five pixels.

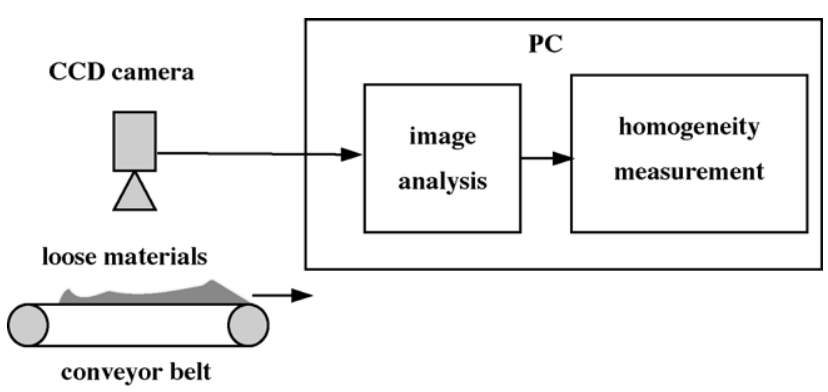

Fig. 1. Schematic diagram of the solid mixture analysing set-up. 


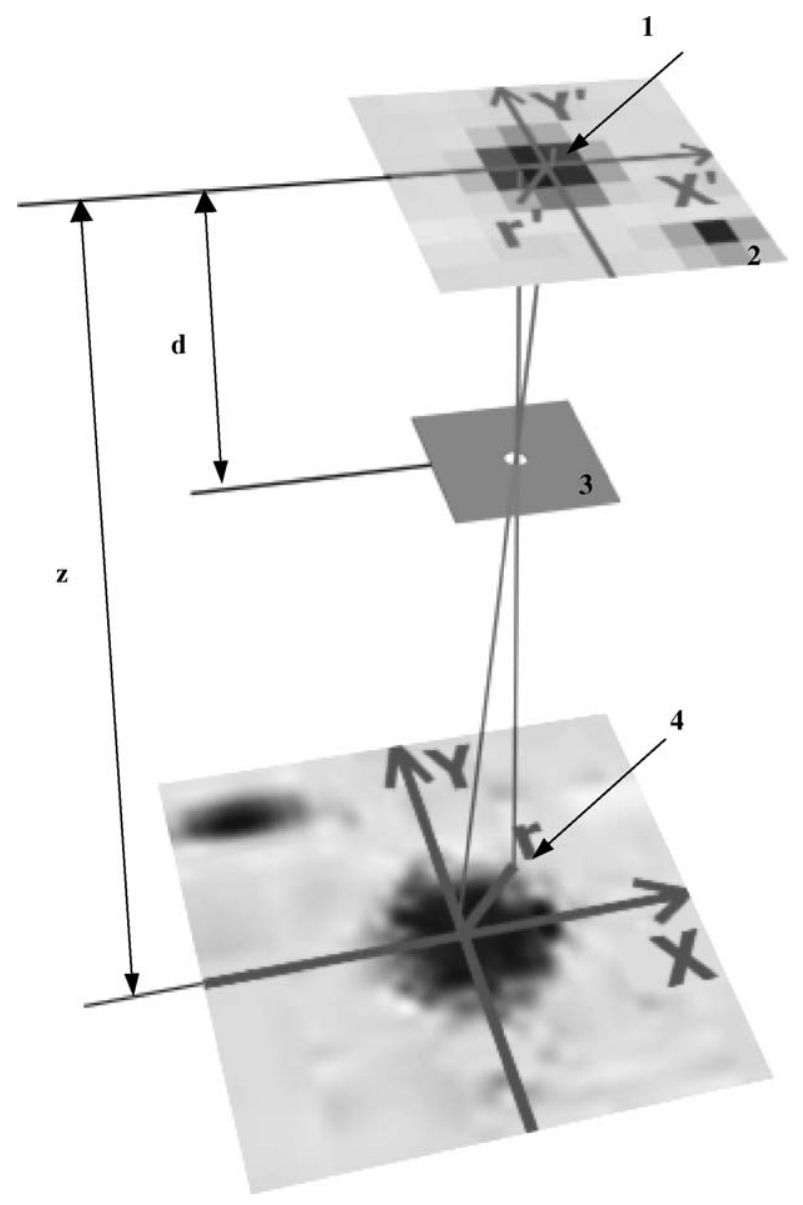

Fig. 2. Pinhole camera model (1, image coordinates; 2 , image plane; 3 , pinhole camera; 4 , world coordinates)

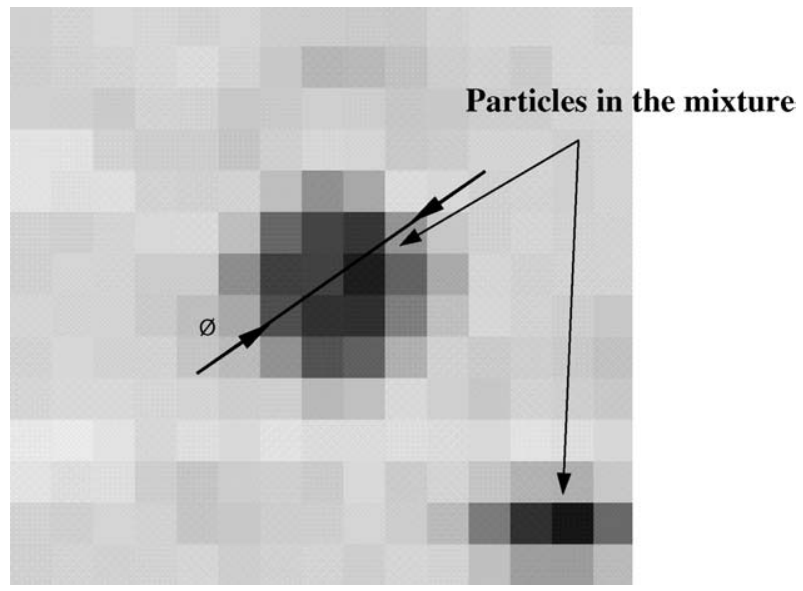

Fig. 3. Example of sampled and quantized binary mixture image.

\section{Homogeneity definition based on PCA}

\subsection{What is PCA?}

Each image transformed as described above consists of rows of pixels describing the solids concentration of the powder stream. Taking into account the rows of an image that comprise information about powder mixture we further assume that the number of such rows is equal to $N$. Typical brightness changes within any row have a probabilistic character, so that they can be treated as any stochastic stationary process $X_{n}(m), n=0, \ldots$, $N-1, m=0, \ldots, M-1$, where $M$ is a number of pixels for each row, and $n$ is a row number. Hence a mathematical model of an image can be defined by a stochastic $N$-dimensional image vector $\mathbf{X}=\left[X_{n}(m)\right], n=0, \ldots, N-1$ where each vector element is called an elementary process (EP). Statistical relationships among EP processes have been determined by a covariance matrix $\mathbf{C}_{\boldsymbol{X}}$ of size $N \times N$, where a matrix term $C_{i j}$ is determined as

$C_{i j}=\frac{1}{M} \sum_{m=0}^{M-1}\left(X_{i}[m] \cdot X_{j}[m]\right), \quad i, j=0, \ldots N-1$

In this, $X_{i}[m], X_{j}[m]$ are denoted as the $\mathrm{EP}_{i}$ and the $\mathrm{EP}_{j}$ processes. Furthermore, we suggest assuming that each EP process is a stationary process and its statistical parameters are constant for single sample.

For defining a homogeneity criterion we propose using the principle component analysis (PCA) of EP processes [15]. The PCA technique, which uses the singular-value decomposition method, detects a ratio correlation among the EPs processes. The PCA has been chosen for its high efficiency for categorizing and classifying huge data sets such those of EP processes.

The PCA technique consists of finding linear transformations called clouds of data of the original processes $\left\{\mathrm{EP}_{n}\right\}$ $n=0, \ldots, N-1$, that have the property of being uncorrelated. This transformation is done by first normalizing the variables so that they have means of zero and a variance of one. The PCA determines the perpendicular axes (called eigenvectors), which are defined by the dimensions of a covariance matrix. The principal components are the eigenvectors obtained from an eigenvector-eigenvalue decomposition of the covariance matrix of the EP processes. The eigenvalue $\lambda_{r}$ corresponding to an eigenvector represents the amount of variability between EP processes explained by that eigenvector. The eigenvector of the largest eigenvalue is the first principal component. The eigenvector of the second largest eigenvalue is the second principal component and so on. There will be the same number of axes as variables (dimensions). The longest axis is the first principle component $\left(\mathrm{PC}_{1}\right)$, and next major axis is the second principle component $\left(\mathrm{PC}_{2}\right)$. We make a slice through the cloud of EP processes using the $R$-dimensional space defined by $\mathrm{PC}_{r}, r=1, \ldots$, $R$ and project all of the EP processes onto this space, then obtain a $R$-dimensional representation of the EP processes retaining the maximum variation (information) contained in the multivariate data. The space $\mathrm{PC}_{r}$ provides maximum separation between the EP processes. The percentage $c_{r}$ of the variability explained by the $r$ th principal component is:

$c_{r}(\%)=\frac{\lambda_{r}}{\lambda_{1}+\lambda_{2}+\cdots+\lambda_{R}} \times 100$

For analysis of EP processes we choose those of $\mathrm{PC}_{r}$, with the greatest percentages $c_{r}$. 


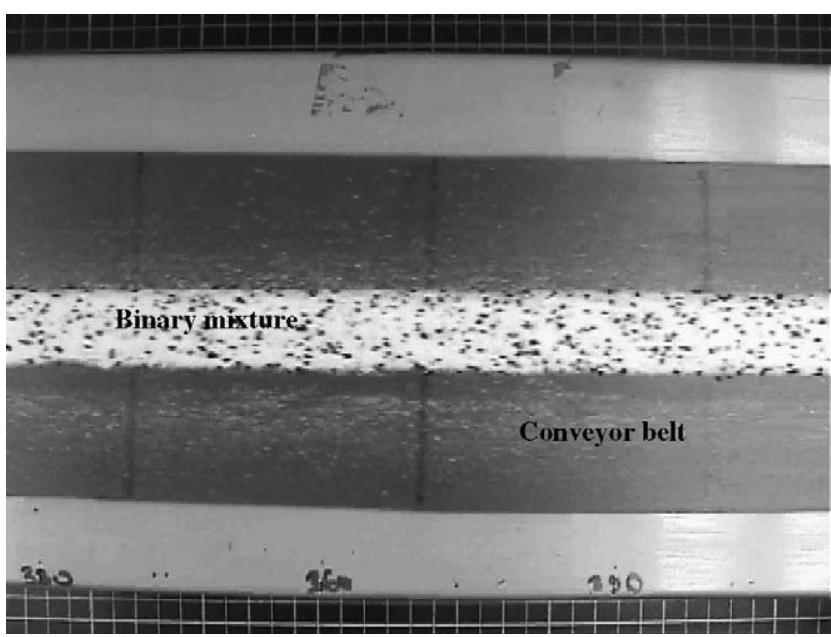

Fig. 4. Photograph of the binary powder layer as seen by the CCD camera.

\subsection{Application of PCA to powder mixtures}

Analysing a solid mixture involves the formation of a powder layer of constant width and thickness which is continuously filmed by a CCD camera. The recorded film is further treated to obtain an unlimited series of frames that represents the surface of the mixture layer (15 frames per second). Fig. 4 shows a typical example frame as captured by our set-up.

Matlab 5.2 was used for image analysis. First, the mixture sample is divided up into $L$ rectangular areas of the same size (Fig. 5b), $L$ value being fixed empirically. Next we perform the
Table 1

Percentages $c_{r}, r=1,2,3$ of the variability explained by first, second and third principal component for some rectangular areas

\begin{tabular}{rlll}
\hline Rectangular area number, $l$ & $c_{1}(\%)$ & $c_{2}(\%)$ & $c_{3}(\%)$ \\
\hline 1 & 97.892 & 1.691 & 0.212 \\
2 & 97.027 & 1.807 & 0.685 \\
3 & 94.871 & 4.211 & 0.483 \\
4 & 97.648 & 1.795 & 0.446 \\
70 & 99.322 & 0.559 & 0.088 \\
80 & 98.205 & 1.107 & 0.488 \\
90 & 99.314 & 0.303 & 0.18 \\
100 & 98.722 & 1.03 & 0.152 \\
\hline
\end{tabular}

PCA analysis for each rectangular area. Area row data (EP processes) enable the calculation of eigenvalues $\lambda_{r}$ and eigenvectors of $\left\{X_{n}(m)\right\} n=0, \ldots, K-1$, where $K$ is the number of rows analysed in a rectangular area.

Then we present EP processes of analysed areas onto threedimensional (3D) space. To do this, we choose $\mathrm{PC}_{r}, r=1,2$, 3 only because they ensure the percentage $c_{r}$ of the variability greater than $98 \%$. The example values of $c_{r}$ for three principal components are presented in Table 1. In Fig. 5c, examples of the distributions of EP processes into the 3D space for two rectangular areas are also depicted.

A mixture can be defined as homogenous if any test element of the mixture has the same composition and properties as any other. In our approach, we use rectangular areas of analysed mixture sample as mixture test elements (scale of scrutiny of the mixture).

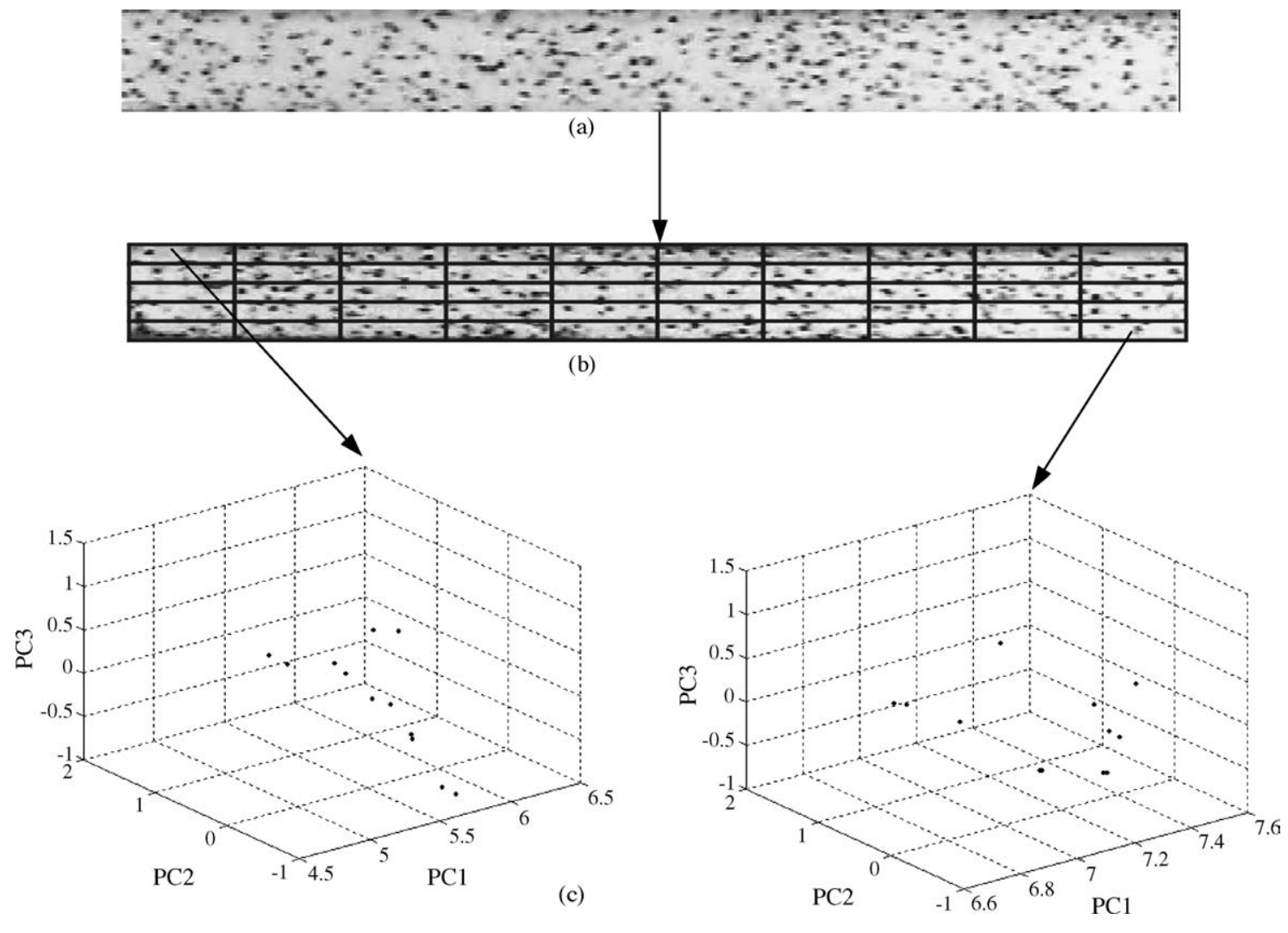

Fig. 5. (a) Mixture sample no. 125; (b) rectangular areas, $L=50$; (c) representation of EP processes into 3D space for first and fiftieth rectangular area. 
For the definition of a criterion of homogeneity of a powder mixture in any frame, we first compare each rectangular area of the analysed mixture sample to another using the similarity index $s_{l}$ which we define as

$$
\begin{aligned}
s_{l}= & \frac{1}{L \cdot K} \sum_{j=1}^{L} \sum_{i=1}^{K} \\
& \times \sqrt{\left(\mathrm{PC}_{1}^{p i}-\mathrm{PC}_{1}^{j i}\right)^{2}+\left(\mathrm{PC}_{2}^{p i}-\mathrm{PC}_{2}^{j i}\right)^{2}+\left(\mathrm{PC}_{3}^{p i}-\mathrm{PC}_{3}^{j i}\right)^{2}} .
\end{aligned}
$$

In the above, $l=1, \ldots, L ; l \neq j, K$ is the number of rows in the analysed rectangular areas, $L$ the number of areas of the mixture sample and $\mathrm{PC}_{k}^{j i}$ is the $k$ th principle component calculated for $i$ th row in $j$ th rectangular area. Comparing rectangular areas is fast and easy by PCA which is an important feature with respect to other methods cited in the Section 1 of this paper.

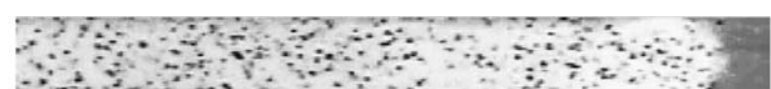

(a)

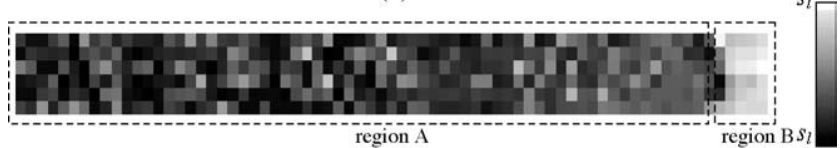

(b)

Fig. 6. (a) Mixture sample no. 104; (b) similarity indexes as grey scale values of a) $(L=426)$.

We confirm that the $s$ value is smaller for similar rectangles and higher when differences between them are greater. For an ideal homogeneous mixture s all rectangular areas are identical and $s_{l}$ calculated for every area is equal to 0 . In Fig. $6 \mathrm{~b}$, similarity indexes $s_{l}$ are presented for the mixture sample no. 104 as grey scale images. They were rescaled to range $[0, \ldots, 1]$. For instance, in Fig. 6a region $\mathrm{A}$ is more homogeneous than region B.

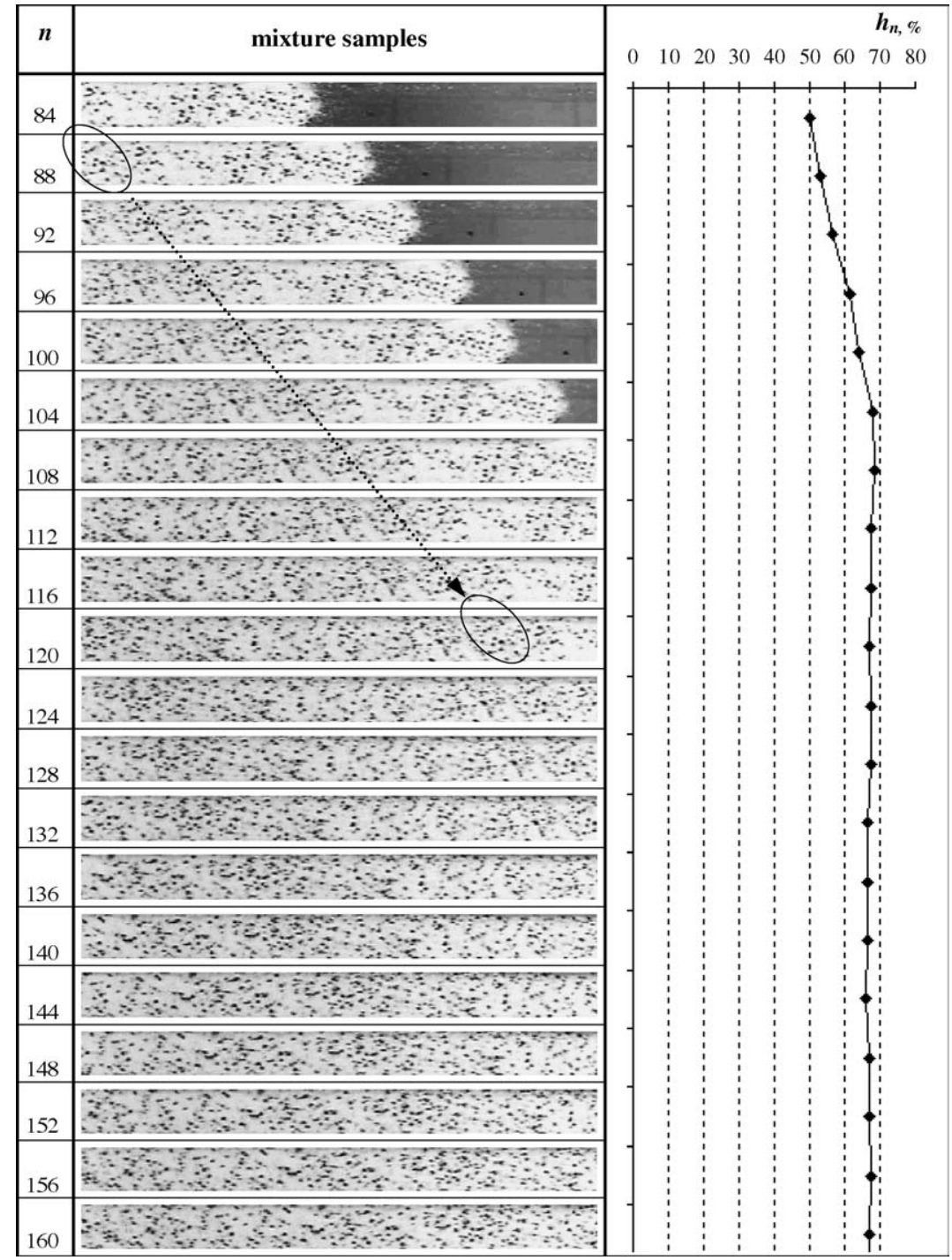

Fig. 7. Mixture samples and calculated homogeneity ratios $(L=100)$, showing a small structural default (circle line). 
Then we calculate the sum $S$ of the $s_{l}$ values specified for all rectangular areas of $n$th mixture sample:

$S_{n}=\sum_{l=1}^{L} s_{l}[n]$.

Finally for $n$th mixture sample, we define a homogeneity criterion $h_{n}$ as

$h_{n}(\%)=\left(1-\frac{S_{n}}{S_{0}}\right) \times 100$.

In Eq. (5), $S_{0}$ is a sum of $s_{l}$ values which are calculated for the perfectly segregated mixture sample, which is used as heterogeneity pattern.

\section{Experimental results}

The loose material transported on the conveyor belt and captured by the CCD camera arrangement was analysed in terms of the above homogeneity criterion (Eq. (5)). Fig. 7 presents the results for 21 mixtures corresponding to the motion of the belt.

As it can be seen in Fig. 7, a part of conveyor belt does not contain any particle of the mixture (samples from 84 to 104). The samples ranging from no. 108 to 164 correspond to a mixture as leaving a continuous mixer, or at the discharge of a batch mixer. The part of conveyor belt without a mixture can be interpreted as area of one mixture component, therefore it disturbs homogeneity of the tested mixture. The value of the homogeneity ratio is the lowest for 84th mixture sample and it increases for the following samples because the part of the belt without mixture becomes smaller.

To calculate a homogeneity ratio we first choose a rectangular area numbered $L$. The single rectangular area should represent quality of the mixture and its size must be adapted to the dimensions of the loose material particles and to a specified scale of scrutiny. Choice of rectangular area number is important because measurement results are dependent on it. Homogeneity ratio values calculated for different $L$ values are presented in Fig. 8. The homogeneity ratio decreases when $L$ decreases, meaning that mixtures are always worse when analysed at a smaller scale of

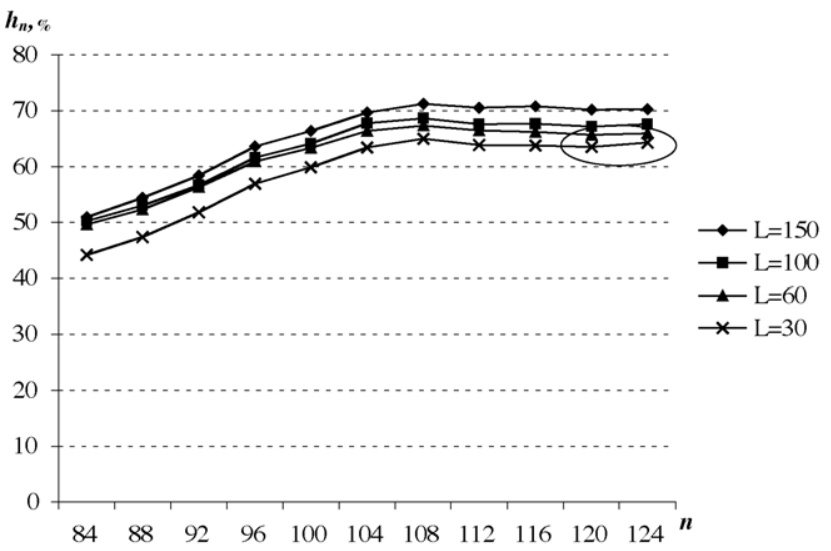

Fig. 8. Homogeneity ratios calculated for different rectangular area number $L$, showing the effect of the small structural default (circle line). scrutiny, as common sense would suggest. Indeed, the intensity of segregation (variance) of a random mixture is known to be inversely proportional to the number of particles per sample. Furthermore, one can also focus on a small area that contains practically no coloured particles and located in the middle of sample no. 96. This area disappears for sample no. 124 and can only be detected for the three lowest $L$ values $(L=30-100)$ in Fig. 7 by a small rise of homogeneity. For the highest value of $L$, the scale of scrutiny is much higher than the scale of segregation (that of the segregated area) and nothing can be detected. This seems to indicate that the methodology employed is sensitive to the structure of the mixture, so that a structural defect can be detected and perhaps withdrawn from the production line.

\section{Concluding remarks}

This paper presents a methodology based on principle component analysis for defining and characterising powder mixture homogeneity as it flows out of a continuous mixer or at the discharge of a batch process. Image analysis is used here and seems to be well suited to illustrate the method, but the method can be applied to other measuring techniques (electrical capacitance, NIR, etc.).

Images of a mixture of black and white particles of semolina transported on a conveyor belt are taken by CCD camera. These are treated with a classical pinhole model and digitized on a grey scale to give information about the concentration of black particles. Each image is considered to be a series of rows of pixels. Each row is treated as a stochastic process. PCA methodology leads to a three-dimensional representation of the processes retaining the maximum variation (information) contained in the multivariate row data. The similarity index values, which are derived from this data treatment, allow the calculation of a criterion of mixture homogeneity.

The mixture is decomposed into rectangular areas whose size corresponds to the scale of scrutiny. First results show that the homogeneity criterion is dependent on the scale of scrutiny chosen. The method allows the detection of small structural defaults in the mixture - that may lead to rejection of part of a production - in an easier way than other types of analysis (auto-correlation or phase portrait).

The use of image analysis illustrates the concepts of scales of segregation and scrutiny, as well as being relatively easy to perform in real-time with few calibration procedures. However, the sensitivity should be verified if it is to be used for industrial applications. Because of the complex phenomena at play and the diversity of powder properties, there is no doubt that no single technique will be able to treat all kinds of powder blend. Therefore, focussing on the development of a range of different techniques and trying to define their domain of applicability for a single and sensitive methodology for data treatment (such as PCA) should be encouraged.

\section{Appendix}

The pinhole camera consists of a non-transparent plane with a small hole. The model used is equivalent to simply describing 
the image formation with such a lens system. The image plane is located parallel to the plane of the camera at a distance $d$. Light radiated by the mixture of loose material passes through the hole and illuminates the image plane.

The relationship between world co-ordinates $(x, y)$ on the initial frame and image co-ordinates $\left(x^{\prime}, y^{\prime}\right)$ on a plane situated at the distance $z$ is as follows:

$x^{\prime}=-\frac{x \cdot d}{z-d} \quad$ and $\quad y^{\prime}=-\frac{y \cdot d}{z-d}$.

Using Eq. (6) it is easy to prove that the relationship between a length $r$ in world co-ordinates and the corresponding length $r^{\prime}$ in image co-ordinates is:

$r^{\prime}=\frac{d \cdot r}{|z-d|}$

From Eq. (7) it follows that $r^{\prime}$ is dependent on $r$ but also on distances $z$ and $d$.A typical image formation model can be described by a continuous image function $g\left(x^{\prime}, y^{\prime}\right)$, whose value corresponds to the brightness at image points [16].

$g\left(x^{\prime}, y^{\prime}\right)=\int_{x} \int_{y} h\left(x^{\prime}-x, y^{\prime}-y\right) \cdot f(x, y) \mathrm{d} x \mathrm{~d} y$

where $f(x, y)$ represent the radiant intensity of the surface of an object and $h\left(x^{\prime}-x, y^{\prime}-y\right)$ is the point spread function.

The point spread function reflects the response of the imaging device to a single point source. Indeed, this function takes into account the effect that objects lose their sharpness in the image.

Brightness at the image point $\left(x^{\prime}, y^{\prime}\right)$ depends on the radiance emitted by the surface of an object in the scene. It is determined by two factors: the illumination falling on the surface and illumination reflected by the surface of the object, which is dependent on its material properties (e.g. reflectance). In our experiments illumination reflected by the mixture depends on the properties of its components. It directly affects the brightness of the image points, therefore we can use images as a data source to analyse solid concentration.

To make a digital image from a continuous-tone image, it must be divided up into individual points of brightness. The process of breaking up a continuous-tone image is referred to as sampling. The 2D continuous image $g\left(x^{\prime}, y^{\prime}\right)$ is divided into $N$ rows and $M$ columns. The intersection of a row and a column is termed a pixel. The appropriate digital brightness data value is assigned to the integer coordinates $[n, m]$ with $n=0, \ldots$, $N-1$ and $m=0, \ldots, M-1$ is $p[n, m]$. The quality of a digital image is directly related to the number of pixels and the range of brightness values. A continuous image function $g\left(x^{\prime}, y^{\prime}\right)$ can be sampled using a discrete grid of sampling points $x=n \Delta, y=m \Delta$ in the plane. Two neighbouring sampling points are separated by distance $\Delta$ along $x$ axis and along $y$ axis. The ideal sampling $s\left(x^{\prime}, y^{\prime}\right)$ in the regular grid can be represented using a collection of Dirac distributions:

$s\left(x^{\prime}, y^{\prime}\right)=\sum_{n=0}^{N-1} \sum_{m=0}^{M-1} \delta\left(x^{\prime}-n \Delta, y^{\prime}-m \Delta\right)$

where $\Delta$ is the sampling distance or interval and $\delta(\cdot, \cdot)$ is the ideal impulse function. The sampled image $g_{s}[n, m]$ is then the product of the continuous image $g\left(x^{\prime}, y^{\prime}\right)$ and the sampling function $s\left(x^{\prime}\right.$, $\left.y^{\prime}\right)$ :

$g_{s}[n, m]=g\left(x^{\prime}, y^{\prime}\right) \cdot \sum_{n=0}^{N-1} \sum_{m=0}^{M-1} \delta\left(x^{\prime}-n \Delta, y^{\prime}-m \Delta\right)$

The process of determining digital brightness values ranging from black, through grey tones, to white is referred to as quantization. The combination of sampling and quantization processes is referred to as image digitization.

\section{References}

[1] J. Gyenis, Assessment of mixing mechanisms on the basis of concentration patterns, Chem. Eng. Process. 38 (1999) 655-674.

[2] R. Weinekötter, H. Gericke, Mixing of Solids, Particle Technology Series, Kluwer Academic Publishers, Dordrecht, 2000.

[3] P. Danckwerts, Theory of mixtures and mixing, Research (London), 6, 1953, 355-361.

[4] F.J. Muzzio, P. Robinson, C. Wightman, D. Brone, Sampling practices in powder blending, Int. J. Pharm. 155 (1997).

[5] C.F. Harwood, R. Davies, M. Jackson, E. Freeman, An optic probe for measuring the mixture composition of powders, Powder Technol. 5 (1971/1972) 77-80.

[6] O. Berntsson, L.G. Danielsson, B. Lagerholm, S. Folestad, Quantitative in-line monitoring of powder blending by near infrared reflection spectroscopy, Powder Technol. 123 (2002) 185-193.

[7] R. Weinekötter, L. Reh., Characterisation of particulate mixtures by inline measurements, Particle Particul. Syst. Charact. 11 (1994) 284-290.

[8] D. Steinmetz, B. Hocine, M. Poux, C. Laguérie, Analyse du mélange de poudres en fluidisation gazeuse à l'aide de la méthode de défluidisation et par capteur à fibre optique, Powder Technol. 89 (1996) 139-147.

[9] C.K. Lai, D. Holt, J.C. Leung, C.L. Clooney, G.K. Raju, P. Hansen, Real-time non-invasive monitoring of dry powder blend homogeneity, AIChE J. 47 (11) (2001) 2618-2622.

[10] T. Dyakowski, L.F.C. Jeanmeure, A.J. Jaworski, Applications of electrical tomography for gas-solids and liquid-solids flows - a review, Powder Technol. 112 (2000) 174-192.

[11] K.L. Ostrowski, S.P. Luke, M.A. Benett, R.A. Williams, Application of electrical capacitance tomography for on-line and off-line analysis of flow pattern in horizontal pipeline of pneumatic conveyer, Chem. Eng. J. 77 (2000) 43-50.

[12] W. Warsito, L.S. Fan, Measurement of real-time flow structures in gas-liquid and gas-liquid-solid flow systems using electrical capacitance tomography, Chem. Eng. Sci. 56 (2001) 6455-6462.

[13] N. Ehrhardt, M. Montagne, H. Berthiaux, B. Dalloz-Dubrujeaud, C. Gatumel, Assessing the homogeneity of powder mixtures by on-line electrical capacitance, Chem. Eng. Process. 44 (2005) 303-313.

[14] S. Muerza, H. Berthiaux, S. Massol-Chaudeur, G. Thomas, A dynamic study of static mixing using on-line image analysis, Powder Technol. 128 (2002) 195-204.

[15] B.R. Horn, Robot Vision, MIT Press, 1986.

[16] D.F. Morrison, Multivariate Statistical Methods, McGraw-Hill Book Company, 1976. 